\title{
Parameter Selection and Stimulating Effects of an Adjustable Gastric Electrical Stimulator in Dogs
}

\author{
Xiaojuan Guo • Yanmei Li • Shukun Yao • Shaoxuan Chen • \\ Yuhui Du $\cdot$ Zhihua Wang
}

Published online: 19 July 2013

(C) The Author(s) 2013. This article is published with open access at Springerlink.com

\begin{abstract}
Background Gastric electrical stimulation (GES) has been proposed as a promising therapeutic option in treating obesity for 20 years. Currently, the available device of GES cannot meet the clinical needs. The purpose of this study is to verify the effect of a new type of adjustable gastric electrical stimulator in reducing food intake and body weight. Methods Eight beagle dogs randomly followed GES and sham GES for 3 months in a crossover design. Parameters were adjusted and individualized during the experiment. Symptoms of GES were recorded, and the effective parameters were selected. Resistance to GES was assessed. Food intake and body weight were measured to evaluate the effect of GES.

Results The effective parameters were varied among the dogs. Resistance to GES was observed in different periods in dogs. Parameters needed to be adjusted every $10.2 \pm 2.1$ days during the period of GES. Food intake during GES for 3 months was significantly reduced than that during sham GES of 3 months $(P<0.05)$. With the decreased food intake, body weight was
\end{abstract}

X. Guo $\cdot$ S. Yao $(\bowtie)$

Graduate School, Peking Union Medical College and Chinese

Academy of Medical Sciences,

9 Dong Dan San Tiao, Dongcheng District, Beijing 100730,

People's Republic of China

e-mail: yaoshukun6@yahoo.com.cn

X. Guo $\cdot$ Y. Li $\cdot \mathrm{S}$. Yao $\cdot \mathrm{S}$. Chen

Department of Gastroenterology, China-Japan Friendship Hospital,

2 Yinghua East Road, Chaoyang District, Beijing 100029,

People's Republic of China

Y. Du

Noted Technology Development Co. Ltd, 7 Gao Xin Nan,

Nanshan District, Shenzhen 518057, People's Republic of China

Z. Wang

Institute of Microelectronics, Tsinghua University,

30 Shuangqing Road, Haidian District, Beijing 100084,

People's Republic of China significantly reduced by the end of GES of 3 months compared with that of sham GES of 3 months $(P<0.05)$.

Conclusions Food intake and body weight of dogs are significantly reduced by adjustable GES. Individual parameters and resistance during GES are required to be considered. The new adjustable device may have good prospects of clinical application for obesity.

Keywords Gastric electrical stimulation · Individual parameter $\cdot$ Resistance $\cdot$ Obesity

\section{Introduction}

Obesity is prevalent worldwide. Various treatments are available for obesity, such as exercise, diet, drugs, and surgery. However, there is a lack of ideal therapy for obesity [1,2]. Gastric electrical stimulation (GES) has been considered as a promising therapeutic option in treating obesity for approximately 20 years [3-5]. This technique is performed through two electrodes inserted into the distal stomach and connected to a stimulator implanted in the abdominal wall. By delivering electrical pulses to the stomach, GES was reported to alter gastric motility, inhibit appetite, and reduce body weight [6-8]. The gastrointestinal anatomy is not affected during the procedure of GES. GES is less invasive and a safer method for treating obesity compared with bariatric surgery [9-11].

There are several electrical stimulation methods which involve long pulse stimulation, short pulse stimulation, and stimulation with trains of pulses [12, 13]. Currently, GES with trains of short pulses is mainly used in the clinical study for obesity $[14,15]$. These studies have been confined to the use of GES with the following typical parameters: a pulse width of $0.3 \mathrm{~ms}$, an amplitude of 5-10 mA, a frequency of $40 \mathrm{~Hz}$, and $2 \mathrm{~s}$ on $-3 \mathrm{~s}$ off. The commercial implantable devices of short pulses used in clinical studies have been 
mainly adapted from a cardiac or nerve stimulator [15-18]. After intensive investigation of GES, some of the following problems must be considered: (1) Although the pulse width $<1 \mathrm{~ms}$ is enough for cardiac or nerve stimulation, short pulses for GES may not be potent enough [19, 20]. (2) Based on differences of individual sensitivity, adjustable parameters are required in a wide range [21, 22]. (3) A fixed mode of parameters is insufficient and not adequately maintained for long-term use.

In collaboration with the Institute of Microelectronics of Tsinghua University, we have developed a new type of adjustable gastric electrical stimulator. This stimulator features a pulse width of $0.3-10 \mathrm{~ms}$, an amplitude of $1-10 \mathrm{~mA}$, a frequency of $40 \mathrm{~Hz}$, and $2 \mathrm{~s}$ on- $3 \mathrm{~s}$ off. The parameters of this device are adjustable in line with stimulation, and the battery can be remotely recharged.

In this study, a type of adjustable stimulator was applied to produce chronic GES on beagle dogs. Parameters were adjusted and individualized according to demand during GES. Resistance (i.e., diminution in the response to GES after long-term use) was assessed. Food intake and body weight were measured to evaluate the effect of GES.

\section{Methods and Procedures}

\section{Animal Preparation and Surgical Procedure}

Eight healthy female beagle dogs (Academy of Military Medical Sciences) $(8.5-13 \mathrm{~kg})$ were involved in this study and allowed an acclimation period of 2 weeks. After an overnight fast, the dogs were anesthetized with a combination of fentanyl $(2 \mu \mathrm{g} / \mathrm{kg})$, propofol $(2 \mathrm{mg} / \mathrm{kg})$, rocuronium $(0.6 \mathrm{mg} / \mathrm{kg})$ and maintained on $1-2 \%$ sevoflurane in oxygen $(1.0 \mathrm{~L} / \mathrm{min})$ carrier gases delivered from a ventilator after endotracheal intubation. Vital signs (respiratory rate, pulse, and tongue color) were monitored. By laparoscopy, one pair of platinum-iridium electrodes was sutured into the seromuscular layer along the greater curvature of the stomach, $3.0 \mathrm{~cm}$ above the pylorus. The electrodes were $1.0 \mathrm{~cm}$ apart. An adjustable electrical stimulator (Noted Technology Development Co. Ltd., China) was embedded under the costal margin of the right upper quadrant subcutaneously and then secured in place by a purse-string suture. After surgery, the dogs wore special jackets and were allowed to recover in their individual cages. The room was humidity and temperature controlled $\left(20-22{ }^{\circ} \mathrm{C}\right)$ with a fixed 12-12-h light-dark cycle. The dogs had enough solid food at a set time (5-7 pm daily) and had free access to water in their home cages at all times. The postoperative situations including swelling, secretions, ulcer, subcutaneous transposition, wound healing, and exposed material were observed and recorded. All experiments were performed after the dogs were completely recovered, usually 2 weeks after the surgery.
The study was approved by the Animal Care and Use Committee of China-Japan Friendship Hospital.

\section{Symptoms}

During the experiment, the situations of dogs including spirit, vital signs, activities, and defecation frequency were recorded. Evaluation of the dogs' symptoms included licking with the tongue, sialorrhea, vomiting, yawning, barking, groaning, belching, murmuring, and dysphoria. These symptoms were assessed based on their frequency and/or severity ( 0 : never; 1 : seldom/mild; 2: often/moderate; 3: continues/severe). The total symptom score of each dog was derived during the experiment, and its correlation with the stimulation energy was assessed. Based on our preliminary acute animal experiment, resistance to GES was demonstrated. Symptoms were recorded daily to explore the feature of resistance and to adjust parameters. To make the evaluation objective, the person who evaluated the signs was objective and blinded from the study design.

\section{Selection of Stimulation Parameters}

The subcutaneous stimulator received stimulation signals which were programmed by an external programmer during the experiment. The periodic rectangular pulses with an amplitude of 1-10 mA, a pulse width of $0.3-$ $10 \mathrm{~ms}$, a frequency of $40 \mathrm{~Hz}$, and $2 \mathrm{~s}$ on $-3 \mathrm{~s}$ off were delivered. After being completely recovered from the surgery, each dog endured a selection period of pulse width for 2 weeks, generally from small to large $(0.3,0.75$, $1,2,3,4,5,6,7,8,9$, and $10 \mathrm{~ms})$, and simultaneously, the other parameters were fixed relatively. During the selection period, GES was applied for a whole day except that symptom score was $\geq 3$.

The selection of stimulation parameters was based on the total symptom score. Intolerable parameters were recognized when the total symptom score was $\geq 3$. The maximum pulse widths were selected which did not induce obvious symptoms (score $<3$ ). If the intolerable pulse width was $\leq 1.0 \mathrm{ms,}$ lower amplitude was chosen (3, 6, and $9 \mathrm{~mA})$.

\section{Food Intake and Weight}

During the whole study period, food intake was measured daily, and fasting weight was recorded weekly. The dogs were given $>500 \mathrm{~g}$ of food (laboratory diet, protein $23.8 \%$, fat $8.4 \%$, fiber $2.9 \%, 3.54 \mathrm{kcal} / \mathrm{g}$ ). Food was provided at a set time slot (5-7 pm). During the experiment, GES or sham GES was practiced $1 \mathrm{~h}$ before food intake and then continued for $3 \mathrm{~h}$. The remaining food was removed at the end of the stimulation period, and daily food consumption was recorded. 


\section{Experimental Protocol}

The experiment followed a crossover trail. After the periods of recovery and selection of the initial effective stimulation parameters, the dogs were randomly studied in two groups: in group A, four dogs underwent a 3-month treatment with GES, a 2-week washout period, and a 3-month control with sham GES sequentially. The other four dogs in group B underwent the experiment in a reversed order. The procedures for sham GES were the same as the GES procedures except that the stimulators were not turned on. Parameters were selected, individualized, and adjusted during the experiment. The system impedance of each stimulator was checked before and after the placement and then every week throughout the study.

\section{Statistical Analysis}

All data were presented as mean \pm SD. Differences in food intake and body weight across GES and sham GES were analyzed by crossover ANOVA. Differences of food intake and body weight within groups were evaluated using repeated measures ANOVA. The level of statistical significance was set at $p<0.05$.

\section{Results}

Symptoms

All dogs completed the study, and none was observed to display severely abnormal behaviors during the application of GES. Symptoms were not observed with sham GES. Symptoms of every dog during higher-intensity GES were different. Frequent symptoms during GES included sialorrhea, vomiting, barking, dysphoria, and licking with the tongue. During lower-intensity GES, dogs did not display severe signs of discomfort. Symptoms were adapted when the symptom score was 1-2. Food intake was affected with mild symptoms during GES. The most intolerable symptoms included continued sialorrhea, vomiting (which were observed in all of the dogs during the higher-intensity GES), barking (in three dogs), and dysphoria (in three dogs). The time for symptoms induced by GES to appear varied among the dogs, ranging from $5 \mathrm{~min}$ to $1 \mathrm{~h}$ after the operation of GES.

\section{Selection of Parameters}

1. Initial effective stimulation parameters (i.e., the maximum pulse widths which did not induce obvious symptoms $($ score $<3)$ ), which were lower than the initial intolerable parameters, were adopted. Meanwhile, food intake could be significantly reduced by the selected parameters.
2. Resistance was recognized as when an increase in food intake after a period of GES was observed with initial effective stimulation parameters. Subsequently, a decrease in food intake was observed when the stimulation intensity was elevated with the extracorporeal programmer.

3. Resistance was induced by chronic GES. Resistance was observed in different durations among the dogs. A higher intensity of GES was given to the dogs every $10.2 \pm 2.1$ days according to demand.

4. Effective and intolerable parameters varied greatly among the dogs. A trend of individualized parameters was clearly observed. The characteristics of GES parameters were summarized in Table 1.

The initial effective stimulation parameters included the pulse widths of $0.5-1.0 \mathrm{~ms}$ and amplitudes of $3-$ $10 \mathrm{~mA}$ (with the pulse widths and amplitudes adjusted and the other parameters fixed). The initial intolerable parameters included the pulse widths of $0.75-3 \mathrm{~ms}$ and amplitudes of 8-10 mA. The effective stimulation parameters of the later part of GES included the pulse widths of 1-7 ms and amplitude of $10 \mathrm{~mA}$.

5. The effects of stimulation were influenced by the pulse width and amplitude. Dogs were more sensitive to the pulse width than the amplitude. Seven dogs had adapted the pulse width of $0.5 \mathrm{~ms}$ and the amplitude of 3-10 mA at the parameter selection periods of GES. Symptom score was 1-2. When the pulse width increased to $1 \mathrm{~ms}$, the symptom score increased to three in four dogs.

Food Intake

Changes of food intake by GES and sham GES were showed in Table 2. In group A, during the period of GES of 3 months, significant decrease in average food intake was observed with the individualized parameters (GES of 1 month vs. post-surgery, $P<0.05$; GES of 3 months vs. post-surgery, $P<0.05)$. During the period of sham GES of 3 months, food intake showed no significant difference compared with that of post-surgery (sham GES of 1 month vs. post-surgery, $P>0.05$; sham GES of 3 months vs. post-surgery, $P>0.05$ ).

In group B, during the period of sham GES of 3 months, increase in food intake was statistically significant compared with that of post-surgery (sham GES of 3 months vs. postsurgery, $P<0.05)$. During the period of GES of 3 months, significant decrease in average food intake was observed (GES of 1 month vs. post-surgery, $P<0.05$; GES of 3 months vs. post-surgery, $P<0.05)$.

The time profiles for changes on food intake were shown in Fig. 1. The difference in food intake between GES and sham GES was significant. Dogs with GES of 3 months ate significantly less food than those with sham GES of 3 months $(P<0.05)$. Although the fluctuation was observed during GES for 3 months, the trend of decreased food intake obviously 
Table 1 The individual parameters of GES for eight beagle dogs (pulse width (in millisecond)/amplitude (in milliampere))

\begin{tabular}{|c|c|c|c|c|c|c|c|c|}
\hline Number & 1 & 2 & 3 & 4 & 5 & 6 & 7 & 8 \\
\hline Initial effective parameters & $0.5 / 10$ & $0.5 / 3$ & $0.5 / 3$ & $0.5 / 6$ & $0.5 / 4$ & $1.0 / 10$ & $0.5 / 8$ & $0.5 / 5$ \\
\hline Initial intolerable parameters & $3 / 10$ & $1 / 10$ & $2 / 10$ & $0.75 / 10$ & $0.75 / 5$ & $3.0 / 10$ & $1.5 / 10$ & $0.75 / 8$ \\
\hline Effective parameters of the later part & $4.0 / 10$ & $1 / 10$ & $3.0 / 10$ & $4.0 / 10$ & $2.0 / 10$ & $7.0 / 10$ & $4.0 / 10$ & $3.0 / 10$ \\
\hline
\end{tabular}

Both the effective parameters and the intolerable parameters varied greatly among the dogs. A trend in individual variations was observed with respect to the initial effective stimulation intensity, initial intolerable of stimulation intensity, and the intensity of the later part of stimulation

existed. Moreover, dogs with GES ate less than they did after surgery while dogs with sham GES did not modify their overall food intake (group A) and continued to eat more than that after surgery (group B).

\section{Weight Evolution}

In group A, during the period of GES of 3 months, especially in the later part, significant decrease in body weight was observed (GES of 3 months vs. post-surgery, $P<0.05$ ). During the period of sham GES of 3 months, body weight showed no significant difference compared with that of postsurgery (sham GES of 3 months vs. post-surgery, $P>0.05$ ).

In group $\mathrm{B}$, by the end of sham GES of 3 months, body weight was increased significantly compared with that of post-surgery (sham GES of 3 months vs. post-surgery, $P<0.05)$. During the period of GES of 3 months, with the decrease in food intake, body weight was decreased compared with that of post-surgery (GES of 3 months vs. post-

Table 2 Effect of GES and sham GES with individual parameters on food intake (in gram) and body weight (in kilogram)

\begin{tabular}{|c|c|c|c|c|}
\hline & \multicolumn{2}{|l|}{ Group A } & \multicolumn{2}{|l|}{ Group B } \\
\hline & Food intake & $\begin{array}{l}\text { Body } \\
\text { weight }\end{array}$ & Food intake & $\begin{array}{l}\text { Body } \\
\text { weight }\end{array}$ \\
\hline Post-surgery & $279.3 \pm 14.9$ & $11.2 \pm 1.0$ & $214.0 \pm 8.8$ & $8.8 \pm 0.7$ \\
\hline GES $1 \mathrm{~m}$ & $215.5 \pm 29.3 *$ & $10.6 \pm 1.1^{*}$ & $154.3 \pm 13.5^{*}$ & $9.1 \pm 0.5$ \\
\hline GES $3 \mathrm{~m}$ & $206.0 \pm 8.6^{*}$ & $10.4 \pm 1.1^{*}$ & $153.5 \pm 15.0^{*}$ & $7.8 \pm 0.3 *$ \\
\hline Sham GES $1 \mathrm{~m}$ & $278.8 \pm 22.1$ & $11.0 \pm 0.9$ & $219.3 \pm 8.2$ & $8.9 \pm 0.7$ \\
\hline Sham GES $3 \mathrm{~m}$ & $281.8 \pm 23.9$ & $11.5 \pm 0.9$ & $227.0 \pm 9.1 *$ & $9.4 \pm 0.6^{*}$ \\
\hline
\end{tabular}

Changes of food intake and body weight by GES and sham GES. In group A, during the period of GES of 3 months, significant decrease in average food intake and body weight was observed $(P<0.05$ GES of 1 month vs. the post-surgery; $P<0.05$ GES of 3 months vs. the postsurgery). In group B, at the end of sham GES of 3 months, increase in food intake and body weight was significant compared with that of post-surgery ( $P<0.05$ GES of 3 months vs. the post-surgery). At the end of GES of 3 months, significant decrease in average food intake and body weight was observed $(P<0.05$ GES of 3 months vs. the postsurgery)

$* P<0.05$ indicates statistical significance of differences from postsurgery surgery, $P<0.05)$. Changes of body weight by GES and sham GES were shown in Table 2.

The difference on body weight of dogs between GES and sham GES was statistically significant. Body weight by the end of GES of 3 months was obviously decreased compared with that of sham GES of 3 months $(P<0.05)$. GES induced
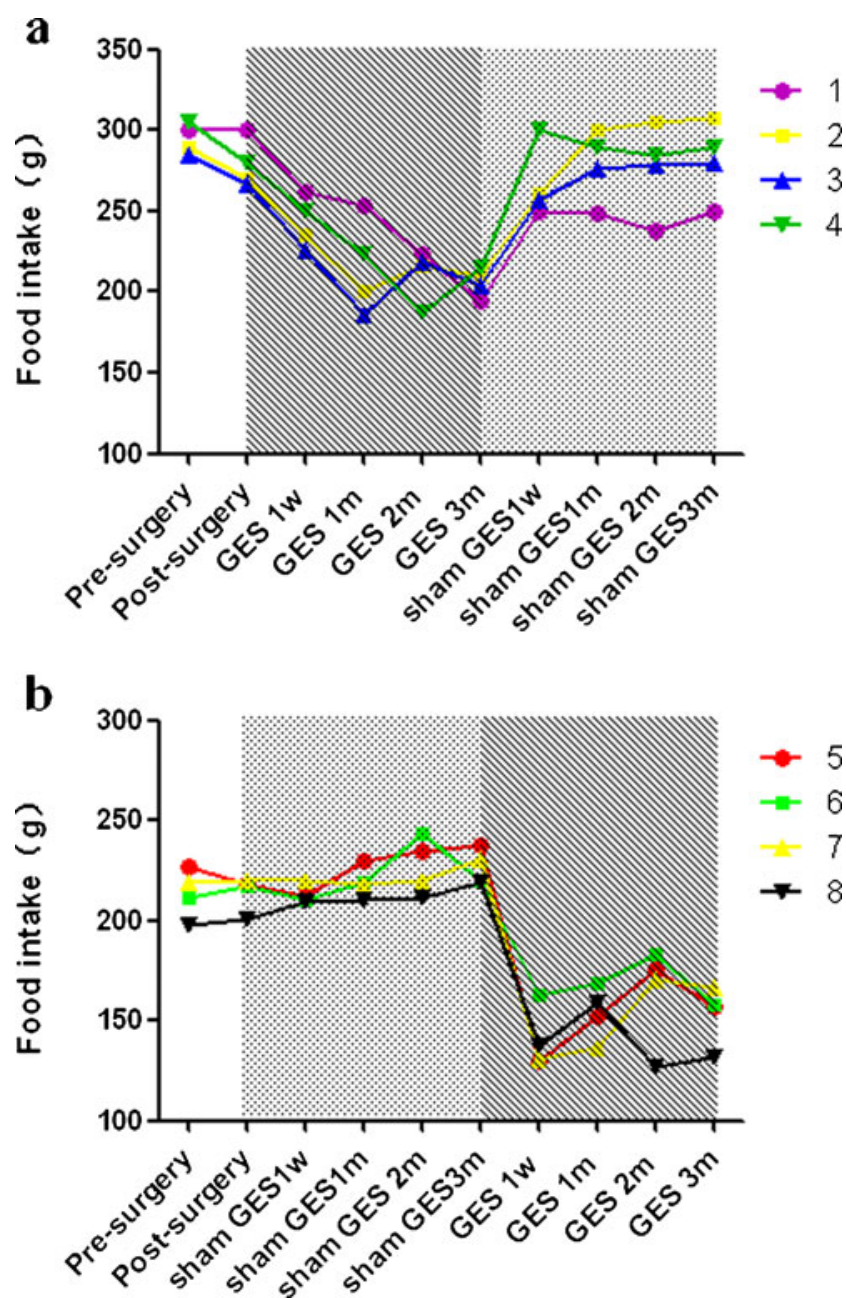

Fig. 1 a Effect of GES and sham GES with individual parameters on food intake of group A (in gram). b Effect of GES and sham GES with individual parameters on food intake of group B (in gram). The difference in food intake between GES and sham GES was significant. Dogs with GES of 3 months ate significantly less food than those with sham GES of 3 months $(P<0.05)$ 

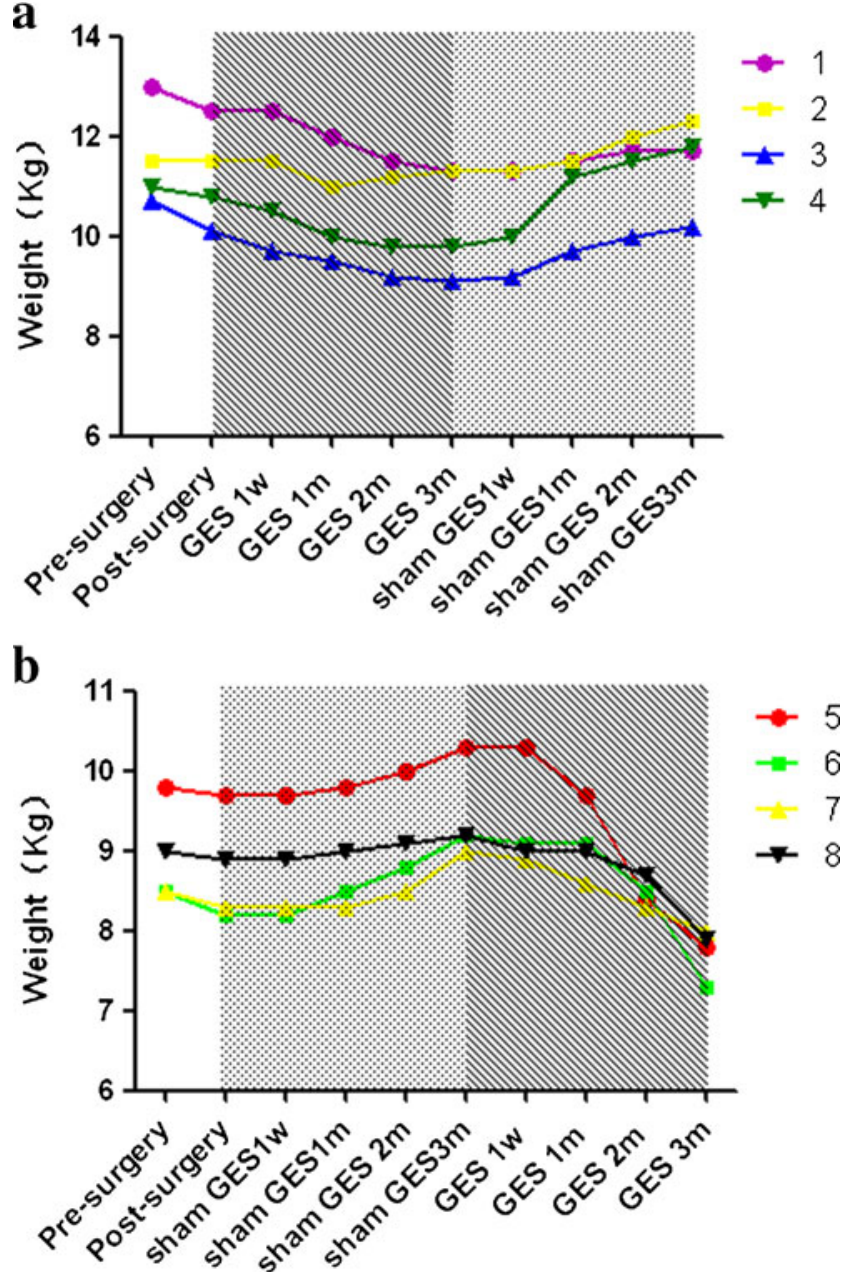

Fig. 2 a Effect of GES and sham GES with individual parameters on body weight of group A (in kilogram). b Effect of GES and sham GES with individual parameters on food intake of group B (in kilogram). The difference in body weight between GES and sham GES was significant. Body weight by the end of GES of 3 months was obviously decreased compared with that of sham GES of 3 months $(P<0.05)$

weight loss while sham GES did not decrease body weight, and the weight even continued to increase gradually during the period of sham GES. The time profiles for changes in body weight were shown in Fig. 2.

During the experiment, no adverse reactions were observed, and the security of GES was indicated. The impedance was in the range of 500-1,000 $\Omega$ during the entire study period. These values were within the normal range of electrode impedance.

\section{Discussion}

In the present study, using a new adjustable device, we found the following: (1) Food intake and body weight of the dogs were reduced significantly after the adjusted stimulation intensity according to demand. (2) The response of dogs to GES varied according to individual dogs. The pulse width of the effective stimulation varied greatly. (3) Using chronic GES, resistance to parameters was observed. Parameters needed to be adjusted every $10.2 \pm 2.1$ days during the period of GES.

GES has been reported to reduce food intake and body weight in animals and in human studies [15, 23]. A study followed up 69 European obese patients and reported significant reductions in weight by GES [24]. However, in some studies, a loss in excess body weight was not observed [25-27]. A clinical trial with 103 patients in the USA reported no greater weight loss at 7 months in those with the device activated compared with those with sham stimulation [27]. The reasons for these differences in results between studies using the same method are unclear.

Recently, more experiments have proved the importance of pulse width in the stimulation process [28-30]. The possible mechanisms between expanded pulse width and reduced food intake or body weight have not been fully elucidated. Pathways of mechanical, neuronal, and hormonal systems have a close relation with GES in the regulation of energy balance [31-33]. Studies have shown that the stomach is composed of smooth muscle cells with a slow response to electrical stimulation and that GES with short pulses may not be potent enough to activate smooth muscles and alter their function [19, 34]. Gastric slow waves were not altered by GES with pulse width shorter than $1 \mathrm{~ms}$ [34]. GES with a pulse width of $2 \mathrm{~ms}$ was found significantly decreased liquid and solid gastric emptying in canines [29]. With an increased pulse width, neuronal responses to GES were strengthened [35]. Moreover, an increase in pulse width of GES had the largest effect on ventral medial hypothalamic neuronal activity, and these effects were paralleled by pulse width-dependent reductions in food intake and body weight [28]. One recent study also reported that expanded pulse width GES increased oxytocin and decreased ghrelin in the hypothalamus of rats [36]. Based on the relationship between weight loss and pulse width, the recommended pulse width would be $>3 \mathrm{~ms}$ [28]. These studies support the notion that GES with an expanded pulse width is an effective treatment for obesity.

Individual parameters of GES have been rarely reported. Data from clinical studies [21, 37] showed that there were individual differences in visceral sensitivity. There was also a wide diversity of visceral responses to GES among individuals. The inhibitory effects of GES on gastric function were correlated with visceral sensitivity of the individual [22]. Optimal parameters should be determined according to the individual sensitivity to GES. Observation and evaluation of visceral sensitivity could be used to screen patients who need a long-term implantable GES. These studies indicated that a fixed pattern of GES is not an ideal adoption, and individual parameters are more effective. In our experiment, the reaction of each dog to chronic GES was different. A 
trend in individual variations was observed in respect to the initial effective stimulation intensity, initial intolerable stimulation intensity, and the intensity of the later part of stimulation. Based on the factor of individual differences, effective parameters were ensured for every dog by selection of parameters. This suggests that to improve the efficacy of GES, the individualized choice of stimulation parameters is important. Our results may explain why GES was ineffective for some patients in previous clinical studies. Our findings also indicate that, in addition to the need for screening parameters for patients in further studies [31], selection and adjustment of parameters should be based on individual symptoms to GES (appetite, satiety, and discomfort) and food intake, to achieve the optional stimulation effect.

In the present study, resistance was observed during GES. In a previous canine study during ten consecutive days, food intake and body weight were reduced by GES (375 ms, $6 \mathrm{cpm}$, and $4 \mathrm{~mA}$ ) within a few days, while some weight was regained during the latter stages of stimulation [38]. During our chronic GES study, stimulation parameters needed to be adjusted every $10.2 \pm 2.1$ days because of resistance. After parameters were adjusted according to demand, inhibitory effects on food intake and body weight were observed by increasing the intensity of stimulation. The initial effective stimulation pulse widths were 0.5 to $1.0 \mathrm{~ms}$. The intensity of stimulation was gradually increased with resistance. The effective stimulation pulse widths of the later part of GES were 1.0 to $7.0 \mathrm{~ms}$. These pulse widths were wider than the $0.3 \mathrm{~ms}$ applied in a previous clinical commercial device [19]. Especially with prolongation of the stimulation time and the appearance of resistance, a pulse width much greater than $0.3 \mathrm{~ms}$ is required to inhibit feeding. These data may explain why the use of GES with a pulse width of $0.3 \mathrm{~ms}$ could not achieve the desired effects in all of the patients. They also indicate that the effective stimulation intensity for each individual varies. In addition, parameters should be adjusted according to demand in the process of applying GES. Therefore, the fixed stimulation parameter of $<1.0 \mathrm{~ms}$ pulse width for some individuals may be far less than the effective stimulation intensity required.

A previous study reported that application of long pulse retrograde gastric electrical stimulation (1.1 times the natural frequency) reduced food intake and influenced the regularity of gastric myoelectrical activity [39]. Obvious symptoms were caused by high-intensity stimulation (550-950 ms, 6-9 mA), while no obvious symptoms were caused by low-intensity stimulation (550 ms, 3-4.5 mA). In the present study, sialorrhea and vomiting were induced by high-intensity GES, and no symptoms or otherwise mild symptoms were induced by low-intensity GES. Therefore, symptoms have a close relationship with stimulation intensity. In addition, pulse width and amplitude influence the effect of stimulation. Moreover, in our study, the dogs were more sensitive to pulse width than amplitude. This result indicates that if dogs appeared to be intolerable to the parameters, the pulse width should be lowered first. Conversely, if the effect of stimulation is not obvious, the pulse width should be raised first. Amplitude can be relatively fixed. These results provide an experimental basis for selecting GES parameters.

During the period of sham GES of 3 months, food intake and body weight were significantly increased. This may have resulted from postoperative recovery gradually and adaptation after the intervention. Based on the crossover design, the confounding effects of the operation and the factor of time could be ruled out. The inhibitory effects of GES on food intake and body weight were well expressed by this study design.

Our study suggested that there were individual differences in beagle dogs for GES. The effective pulse width of stimulation had an extensive range. Resistance was found in all of the dogs. Intermittent adjustment of stimulation parameters according to demand, usually every $10.2 \pm 2.1$ days, was required for longterm application of GES. Although dogs are rarely obese, the present study showed that food intake and body weight of dogs were reduced by adjusting stimulation parameters and increasing stimulation intensity by demand. These findings provide experimental support for obesity treatment with GES in clinical trials. Furthermore, in previous clinical studies, the time required to reduce weight by GES was usually longer than 1 year. Based on individual and adjustable parameters, the present study indicated that the time required for observation of the effect of GES might be reduced to 3-6 months.

In conclusion, this study may shed light on applying GES in treating obesity. Individual parameters and resistance during GES are required to be considered. Our new adjustable device may have good prospects of clinical application for obesity.

Acknowledgments This work was supported by the National Natural Science Foundation of China (grant no. 81070299) and the Capital Medical Development Foundation of China (grant no. 2009-2018).

Conflict of Interest The authors declared no conflict of interest.

Open Access This article is distributed under the terms of the Creative Commons Attribution License which permits any use, distribution, and reproduction in any medium, provided the original author(s) and the source are credited.

\section{References}

1. Ahima RS. Obesity: much silence makes a mighty noise. Gastroenterology. 2007;132:2085-6.

2. World Health Organization. Obesity and overweight. In: Fact sheet no. $311 \mathrm{http}: / /$ www.who.int/mediacentre/factsheets/fs311/en/ (2011)

3. Cigaina VV, Saggioro A, Rigo VV, et al. Long-term effects of gastric pacing to reduce feed intake in swine. Obes Surg. 1996;6:250-3.

4. Hasler WL. Methods of gastric electrical stimulation and pacing: a review of their benefits and mechanisms of action in gastroparesis and obesity. Neurogastroenterol Motil. 2009;21:229-43. 
5. Bohdjalian A, Prager G, Aviv R, et al. One-year experience with Tantalus: a new surgical approach to treat morbid obesity. Obes Surg. 2006;16:627-34.

6. Shikora SA. Implantable gastric stimulation for the treatment of severe obesity. Obes Surg. 2004;14:545-8.

7. Yin J, Chen JD. Retrograde gastric electrical stimulation reduces food intake and weight in obese rats. Obes Res. 2005;13:1580-7.

8. Ouyang H, Xing J, Chen JD. Tachygastria induced by gastric electrical stimulation is mediated via alpha- and beta-adrenergic pathway and inhibits antral motility in dogs. Neurogastroenterol Motil. 2005;17:846-53.

9. Shikora SA. Implantable gastric stimulation - the surgical procedure: combining safety with simplicity. Obes Surg. 2004;14:S9-S13.

10. Mintchev MP. Gastric electrical stimulation for the treatment of obesity: from entrainment to bezoars - a functional review. ISRN Gastroenterol. 2013;2013:434706. doi:10.1155/2013/434706.

11. Abell TL, Minocha A, Abidi N. Looking to the future: electrical stimulation for obesity. Am J Med Sci. 2006;331:226-32.

12. Bohdjalian A, Ludvik B, Guerci B, et al. Improvement in glycemic control by gastric electrical stimulation (Tantalus) in overweight subjects with type 2 diabetes. Surg Endosc. 2009;23(9):1955-60.

13. Dellon ES, Bozymski EM. Gastric electrical stimulation: "scoping" out new directions. Gastrointest Endosc. 2007;66:987-9.

14. Favretti F, De Luca M, Segato G, et al. Treatment of morbid obesity with the Transcend Implantable Gastric Stimulator (IGS): a prospective survey. Obes Surg. 2004;14:666-70.

15. Yin J, Chen JD. Implantable gastric electrical stimulation: ready for prime time? Gastroenterology. 2008;134:665-7.

16. Chen J. Mechanisms of action of the implantable gastric stimulator for obesity. Obes Surg. 2004;14 Suppl 1:S28-32.

17. D'Argent J. Gastric electrical stimulation as therapy of morbid obesity: preliminary results from the French study. Obes Surg. 2002;12 Suppl 1:21S-5S.

18. Cigaina V. Gastric pacing as therapy for morbid obesity: preliminary results. Obes Surg. 2002;12 Suppl 1:12S-6S.

19. Zhang J, Tang M, Chen JD. Gastric electrical stimulation for obesity: the need for a new device using wider pulses. Obesity (Silver Spring). 2009;17:474-80.

20. Zhang J, Chen JD. Systematic review: applications and future of gastric electrical stimulation. Aliment Pharmacol Ther. 2006;24: 991-1002.

21. Yao SK, Ke MY, Wang ZF, et al. Visceral sensitivity to gastric stimulation and its correlation with alterations in gastric emptying and accommodation in humans. Obes Surg. 2005;15:247-53.

22. Yao SK, Ke MY, Wang ZF, et al. Visceral response to acute retrograde gastric electrical stimulation in healthy human. World $\mathrm{J}$ Gastroenterol. 2005;11:4541-6.

23. Lei Y, Xing J, Chen JD. The effect on gastric tone of gastric electrical stimulation with trains of short pulses varies with sites and stimulation conditions. Dig Dis Sci. 2008;53(8):2066-71.
24. De Luca M, Segato G, Busetto L, et al. Progress in implantable gastric stimulation: summary of results of the European multicenter study. Obes Surg. 2004;14:S33-9.

25. Shikora SA, Bergenstal R, Bessler M, et al. Implantable gastric stimulation for the treatment of clinically severe obesity: results of the SHAPE trial. Surg Obes Relat Dis. 2009;5:31-7.

26. Hoeller E, Aigner F, Margreiter R, et al. Intragastric stimulation is ineffective after failed adjustable gastric banding. Obes Surg. 2006; $16: 1160-5$.

27. Shikora SA. "What are the Yanks doing?" The U.S. experience with implantable gastric stimulation (IGS) for the treatment of obesity-update on the ongoing clinical trials. Obes Surg. 2004;14:S40-8.

28. Zhang J, Maude-Griffin R, Zhu H, et al. Gastric electrical stimulation parameter dependently alters ventral medial hypothalamic activity and feeding in obese rats. Am J Physiol Gastrointest Liver Physiol. 2011;301:G912-8.

29. Song GQ, Hou X, Sun Y, et al. Effects of retrograde gastric electrical stimulation with pulse trains on gastric emptying of solids and plasma hormones in dogs. Am J Surg. 2007;194:122-7.

30. Du P, Li S, O'Grady G, et al. Effects of electrical stimulation on isolated rodent gastric smooth muscle cells evaluated via a joint computational simulation and experimental approach. Am J Physiol Gastrointest Liver Physiol. 2009;297:G672-80.

31. Mizrahi M, Ben Ya'acov A, Ilan Y. Gastric stimulation for weight loss. World J Gastroenterol. 2012;18:2309-19.

32. Gallas S, Sinno MH, Boukhettala N, et al. Gastric electrical stimulation increases ghrelin production and inhibits catecholaminergic brainstem neurons in rats. Eur J Neurosci. 2011;33:276-84.

33. Xu J, McNearney TA, Chen JD. Gastric/intestinal electrical stimulation modulates appetite regulatory peptide hormones in the stomach and duodenum in rats. Obes Surg. 2007;17:406-13.

34. Lin Z, Forster J, Sarosiek I, et al. Effect of high-frequency gastric electrical stimulation on gastric myoelectric activity in gastroparetic patients. Neurogastroenterol Motil. 2004;16:205-12.

35. Qin C, Chen JD, Zhang J, et al. Modulatory effects and afferent pathways of gastric electrical stimulation on rat thoracic spinal neurons receiving input from the stomach. Neurosci Res. 2007;57:29-39.

36. Zhang J, Liu S, Tang M, et al. Optimal locations and parameters of gastric electrical stimulation in altering ghrelin and oxytocin in the hypothalamus of rats. Neurosci Res. 2008;62:262-9.

37. Yao SK, Ke MY, Wang ZF, et al. Retrograde gastric pacing reduces food intake and delays gastric emptying in humans: a potential therapy for obesity? Dig Dis Sci. 2005;50:1569-75.

38. Xing JH, Brody F, Brodsky J, et al. Gastric electrical-stimulation effects on canine gastric emptying, food intake, and body weight. Obes Res. 2003;11:41-7.

39. Chen JZ, Ueno T, Xu X, et al. Reverse gastric pacing reduces food intake without inducing symptoms in dogs. Scand J Gastroenterol. 2006;41:30-6. 